\title{
Dinámica de la producción primaria y valor nutritivo de tres gramíneas tropicales (Melinis minutiflora, Setaria sphacelata y Brachiaria mutica) en tres estados fenológicos
}

\author{
DYNAMICS OF THE PRIMARY PRODUCTION AND NUTRITIVE VALUE OF THREE TROPICAL \\ GRASSES (Melinis minutiflora, Setaria sphacelata AND Brachiaria mutica) \\ IN THREE PHENOLOGICAL STAGES
}

Gilmar López A. ${ }^{1}$, Jimny Nuñez D. ${ }^{1,3}$, Lucrecia Aguirre T. ${ }^{1,2}$, Enrique Flores M. ${ }^{1,2}$

\section{Resumen}

\begin{abstract}
Se evaluó la dinámica de la producción primaria y el valor nutritivo basado en la variación del rendimiento de materia seca, altura de planta, contenido de proteína cruda, fibra cruda y digestibilidad in vitro de materia seca (MS) en tres estados fenológicos (botón floral, inicio de floración y maduración de semilla) de tres especies de gramíneas tropicales: gramalote (Brachiaria mutica), gordura (Melinis minutiflora) y setaria (Setaria sphacelata). El estudio se realizó en la provincia de Oxapampa, región Pasco (Perú) utilizando un diseño estadístico de parcelas divididas para la variable de producción de biomasa aérea y de bloques completamente randomizado para las variables de valor nutritivo del forraje. B. mutica presentó la mayor altura promedio $(97 \mathrm{~cm})$, seguida por $S$. sphacelata $(55 \mathrm{~cm})$ y M. minutiflora $(40 \mathrm{~cm})$. La superioridad en altura de B. mutica se reflejó en la mayor producción de materia seca acumulada (8978 kg MS/ha) comparada con $S$. sphacelata (5755 kg MS/ha) y M. minutiflora (4338 kg MS/ha). B. mutica y $S$. sphacelata mantuvieron promedios similares de proteína cruda $(6.83$ y $7.45 \%)$ y digestibilidad in vitro $(72.36,73.0 \%)$ y superiores a los de $M$. minutiflora $(5.83 \%$ y $69.6 \%$, respectivamente). El análisis fenológico reveló que $S$. sphacelata completa su desarrollo fenológico en menos tiempo ( 7 meses), seguido de $B$. mutica ( 8 meses) y $M$. minutiflora (9 meses). Se recomienda el uso de $B$. mutica y $S$. sphacelata sobre $M$. minutiflora por su mayor capacidad de producción de materia seca y mejor valor nutritivo.
\end{abstract}

Palabras clave: gramínea tropical; valor nutricional; altura de planta; materia seca, proteína cruda; digestibilidad in vitro; estado fenológico

\footnotetext{
${ }^{1}$ Laboratorio de Ecología y Utilización de Pastizales, Universidad Nacional Agraria La Molina, Lima, Perú

${ }^{2}$ Departamento de Producción Animal, Facultad de Zootecnia, Universidad Nacional Agraria La Molina, Lima, Perú

${ }^{3}$ E-mail: jimny_unalm@hotmail.com
}

Recibido: 26 de septiembre de 2017

Aceptado para publicación: 28 de febrero de 2018 
The dynamics of primary production and nutritional value were evaluated based on the variation of dry matter yield, plant height, crude protein content, crude fibre, and in vitro digestibility of dry matter (DM) in three phenological stages (floral bud, start of flowering and seed maturation) of three species of tropical grasses: para grass (Brachiaria mutica), molasses grass (Melinis minutiflora) and South African pigeon grass (Setaria sphacelata). The study was conducted in the province of Oxapampa, Pasco region (Peru) using a statistical design of divided plots for the variable of production of aerial biomass and completely randomized blocks for the nutritional value of the forage. B. mutica had the highest average height $(97 \mathrm{~cm})$, followed by $S$. sphacelata $(55 \mathrm{~cm})$ and $M$. minutiflora $(40 \mathrm{~cm})$. The superiority in height of $B$. mutica was reflected in the highest cumulative dry matter production $(8978 \mathrm{~kg} \mathrm{DM} / \mathrm{ha})$ compared to $S$. sphacelata $(5755 \mathrm{~kg} \mathrm{DM} / \mathrm{ha})$ and $M$. minutiflora (4338 kg DM/ha). B. mutica and S. sphacelata maintained similar crude protein average $(6.83$ and $7.45 \%)$ and in vitro digestibility $(72.36,73.0 \%)$ and greater than those of M. minutiflora (5.83\% and $69.6 \%$, respectively). The phenological analysis revealed that $S$. sphacelata completes its phenological development in less time (7 months), followed by B. mutica ( 8 months) and M. minutiflora ( 9 months). The use of B. mutica and $S$. sphacelata instead of $M$. minutiflora is recommended due to its greater dry matter production capacity and better nutritional value.

Key words: tropical grass; nutritional value; plant height; dry matter, crude protein; in vitro digestibility; phenological state

\section{INTRODUCCIÓN}

El Valle de Oxapampa, Perú, está constituido por extensas áreas de pastos naturalizados y pastos cultivados que se usan para la explotación ganadera. El valle tiene una temperatura promedio de $15.5^{\circ} \mathrm{C}$, precipitación pluvial de $1500 \mathrm{~mm} /$ año y humedad relativa de $92 \%$. La actividad económica está centrada en el campo agropecuario, con énfasis en la ganadería bovina bajo un sistema de producción extensiva, utilizando pastos introducidos adaptados a suelos bien drenados como Melinis minutiflora, Setaria sphacelata y mal drenados como Brachiaria mutica (IUCN, 2016), de tipo arenosos hasta arcillosos con moderada fertilidad (Peters et al., 2010).

S. sphacelata (Zuloaga et al., 2003) es un pasto perenne originario de África tropical, resistente a la sequía y a bajos niveles de fósforo, salinidad y toxicidad por magnesio
(León, 2008), con producciones entre $10 \mathrm{y}$ 25 t/ha/año con fertilización (Alvim et al., 1986; Martins et al., 2004), 7-12\% de proteína, y hasta $19.9 \%$ con altas dosis de fertilización nitrogenada (Llamas et al., 2017) y 50$55 \%$ de digestibilidad (Mas, 2007; Martins et al., 2004). B. mutica es un pasto de crecimiento rastrero que crece en terrenos húmedos o pantanosos, no tolera sequía y se adapta a suelos ácidos o neutros, pero no salinos. Su reproducción es mediante tallos (HarvardDuclos, 1969; Ira, 1979), con una producción entre 5 y $12 \mathrm{t} / \mathrm{ha} / \mathrm{año}$ en secano (Martins et al., 2004) y hasta $17.4 \mathrm{t} / \mathrm{ha} / \mathrm{año} \mathrm{(Alvim} \mathrm{et} \mathrm{al.,}$ 1986), con $10-12 \%$ de proteína y $50-70 \%$ de digestibilidad (Martins et al., 2004). M. minutiflora se desarrolla en suelos con mediana fertilidad (Martins et al., 2004), considerada como especie invasora con potencial para desplazar a otras especies nativas (Hoffmann et al., 2004), con una producción entre 10 y $13 \mathrm{t}$ de $\mathrm{MS} / \mathrm{ha} / \mathrm{año}$, 6-10\% de proteína y $50-55 \%$ de digestibilidad (Peters et al., 2010). 
Las especies tropicales son altamente productivas debido a sus características fisiológicas C4 (Carámbula, 1996) y a las condiciones ambientales, principalmente de precipitación y temperatura (Ramírez et al., 2009; Nuñez, 2017). El contenido de proteína cruda de las gramíneas puede variar entre $3 \%$ en una gramínea tropical y muy madura hasta más de $30 \%$ en etapa vegetativa y aplicando fertilizantes (Trujillo y Uriarte, 2001). La digestibilidad del forraje varía con el estado de madurez, la especie de pasto y el manejo, donde una pastura tropical en estado vegetativo temprano tiene generalmente una alta digestibilidad (33-44\%) (Nuñez, 2017).

A pesar de la importancia que tienen estos pastos en el desarrollo ganadero del lugar, existe poca información acerca de su comportamiento, contenido de proteína cruda, fibra cruda y digestibilidad, que sirvan para dar un uso racional al forraje. En consecuencia, el presente estudio tuvo como objetivo caracterizar la dinámica de la producción primaria de las gramíneas Brachiaria mutica, Melinis minutiflora y Setaria sphacelata.

\section{Materiales y Métodos}

El estudio comprendió un ensayo destinado a evaluar la productividad primaria y otro orientado a estimar las variaciones en valor nutritivo según el avance de su desarrollo fenológico. El estudio se realizó en la provincia de Oxapampa, región Pasco, a 1800 msnm, temperatura promedio de $15.5^{\circ} \mathrm{C}$ (máxima $21^{\circ} \mathrm{C}$, mínima $10^{\circ} \mathrm{C}$ ), precipitación anual de $1500 \mathrm{~mm}$, humedad relativa de $92 \%$ y $\mathrm{pH}$ del suelo entre 4.5 y 6 .

\section{Dinámica de la Productividad Primaria}

Se caracterizó la dinámica de la producción primaria de las gramíneas $B$. mutica, M. minutiflora y $S$. sphacelata durante un ciclo de crecimiento en potreros instalados de aproximadamente tres años de edad, manejados tradicionalmente (sin fertilización), con similares características de exposición, suelo y clima. Se seleccionaron áreas representativas (área donde domina la especie de interés) y uniformes. Se cercaron $400 \mathrm{~m}^{2}$ dentro de cada potrero y por cada especie. $\mathrm{Al}$ inicio de las lluvias se realizó un corte de uniformización a una altura de $10 \mathrm{~cm}$ desde el suelo. Luego se sortearon las áreas que correspondieron a los meses de evaluación.

Las variables evaluadas fueron producción de biomasa aérea, altura de planta, temperatura y humedad del suelo a profundidades de 5, 20 y $35 \mathrm{~cm}$ para obtener un promedio. La biomasa aérea fue muestreada mensualmente utilizando cuadrantes de $1 \mathrm{~m}^{2}$, la vegetación fue cortada a una altura de 10 $\mathrm{cm}$. Las muestras fueron pesadas en verde, luego llevadas a estufa a $60^{\circ} \mathrm{C}$ por 48 horas para determinar la materia seca (MS) utilizando una balanza digital $\left(\mathrm{OHAUS}^{\circledR}\right.$, Ranger 3000) de $3000 \mathrm{~g}$ de capacidad máxima y 0.1 $\mathrm{g}$ de precisión. La altura fue estimada midiendo la longitud de planta desde el ras del suelo a la hoja bandera. La temperatura del suelo se determinó usando geotermómetros, mientras que para la humedad del suelo se utilizó el método gravimétrico. Las muestras de suelos fueron colectadas en los mismos lugares donde se midió la temperatura, utilizando envases de plástico con cierre hermético para evitar la pérdida de humedad. Las muestras se enviaron al laboratorio para determinar el contenido de humedad en estufa a $105^{\circ} \mathrm{C}$ por 24 horas (Martínez, 1971).

Para analizar los datos de producción de forraje se utilizó el diseño estadístico de parcela divididas en bloques completos al azar, donde los bloques representan áreas homogéneas de suelo, las parcelas dentro de bloques a las especies de pastos y las subparcelas los meses del año. Para la comparación de promedios se utilizó la prueba de Duncan con un alpha de 5\%. 


\section{Desarrollo Fenológico y Valor Nutritivo del Forraje}

Se caracterizó el contenido nutritivo en las etapas fenológicas de botón floral, inicio de floración y maduración de semilla. Se utilizaron las mismas áreas y especies forrajeras del primer ensayo y el muestreo se realizó cuando el $70 \%$ de las plantas se encontraban en el estado fenológico indicado. Para obtener muestras para la evaluación nutricional se utilizaron cuadrantes de $1 \mathrm{~m}^{2}$ para cortar la vegetación a $10 \mathrm{~cm}$ de altura, luego se procedió al secado de la muestra a $60^{\circ} \mathrm{C}$ durante 48 horas. Las variables de proteína cruda (PC), fibra cruda y cenizas fueron analizadas utilizando procedimientos estándar (AOAC, 1984), en tanto que la digestibilidad in vitro de la materia seca fue analizada usando la técnica in vitro de dos fases (Tilley y Terry, 1963).

Para el análisis de los datos de valor nutritivo en las distintas etapas fenológicas se utilizó el diseño de bloque completo randomizado con arreglo factorial de $3 \times 3$, donde los factores fueron las tres especies y los tres estados fenológicos, cada uno con dos réplicas. Para comparar los promedios se utilizó la prueba de Duncan con un alpha de $5 \%$.

\section{Resultados y Discusión}

\section{Dinámica de la Producción Primaria}

En las condiciones del estudio, $B$. mutica alcanzó mayor altura en todos los meses de evaluación, alcanzando $184 \mathrm{~cm}$ al finalizar el ciclo de crecimiento, siendo mayor que M. minutiflora y $S$. sphacelata. Este comportamiento se debe principalmente a las características de crecimiento propias de la especie y el rango de humedad del suelo (23.7-30.3\%) más favorable comparado con el suelo de M. minutiflora (21.3-29.3\%) y S. sphacelata (15.3-29.3\%). Cabe resaltar el potencial que muestra $S$. sphacelata para tolerar suelos con baja disponibilidad de agua. Al finalizar el experimento, las especies $M$. minutiflora y $S$. sphacelata alcanzaron similares tamaños ( 91.7 y $96.3 \mathrm{~cm}$, respectivamente). En este estudio, S. sphacelata alcanzó a los seis meses una altura $90.33 \mathrm{~cm}$, valores por debajo a los reportados por Imbaquingo y Naranjo (2010), quienes encontraron una altura promedio de $106 \mathrm{~cm}$ a la misma edad de la planta.

\section{Rendimiento de materia seca}

El rendimiento promedio de materia seca ( $\mathrm{kg} \mathrm{MS} / \mathrm{ha}$ ) varió significativamente entre las especies estudiadas $(\mathrm{p}<0.05)$. B. mutica mostró mayor rendimiento ( $8978 \mathrm{~kg} \mathrm{MS} / \mathrm{ha})$ que S. sphacelata $(5755 \mathrm{~kg} \mathrm{MS} / \mathrm{ha})$ y $M$. minutiflora (4338 kg MS/ha). En los tres primeros meses al inicio de la época de crecimiento (octubre, noviembre y diciembre) se observó un lento desarrollo en todas las gramíneas, lo que es una respuesta general en el ciclo de crecimiento de las plantas forrajeras. Luego, B. mutica mostró el mayor incremento de MS hasta finalizar el ciclo de crecimiento (Figura 1).

El rendimiento de MS muestra una relación directa con la variable altura de planta, lo que muestra que el incremento en altura de planta va acompañado de un incremento en la biomasa estructural (tallos) y foliar (hojas), aunque no se llegó a cuantificar la proporción de tallo y hojas en el presente estudio. Estos resultados muestran el comportamiento productivo de las especies en ausencia de fertilización y suplementación de agua. Estudios en similares condiciones de suelo y clima, sin incorporación de otros elementos como la fertilización y riego reportan producción de MS similares para $B$. mutica $(5 \mathrm{a} 8 \mathrm{t}$ MS/ha) (Bogdan, 1997). Además, para el género Brachiaria se reportan rendimientos de 1.2 a $3.6 \mathrm{t} \mathrm{MS} /$ ha (Costa et al., 1990; Rosemberg et al., 1990) y de $3.53 \mathrm{t} \mathrm{MS/ha} \mathrm{a}$ los 24 días de rebrote en época lluviosa (Cuadrado et al., 2004). 


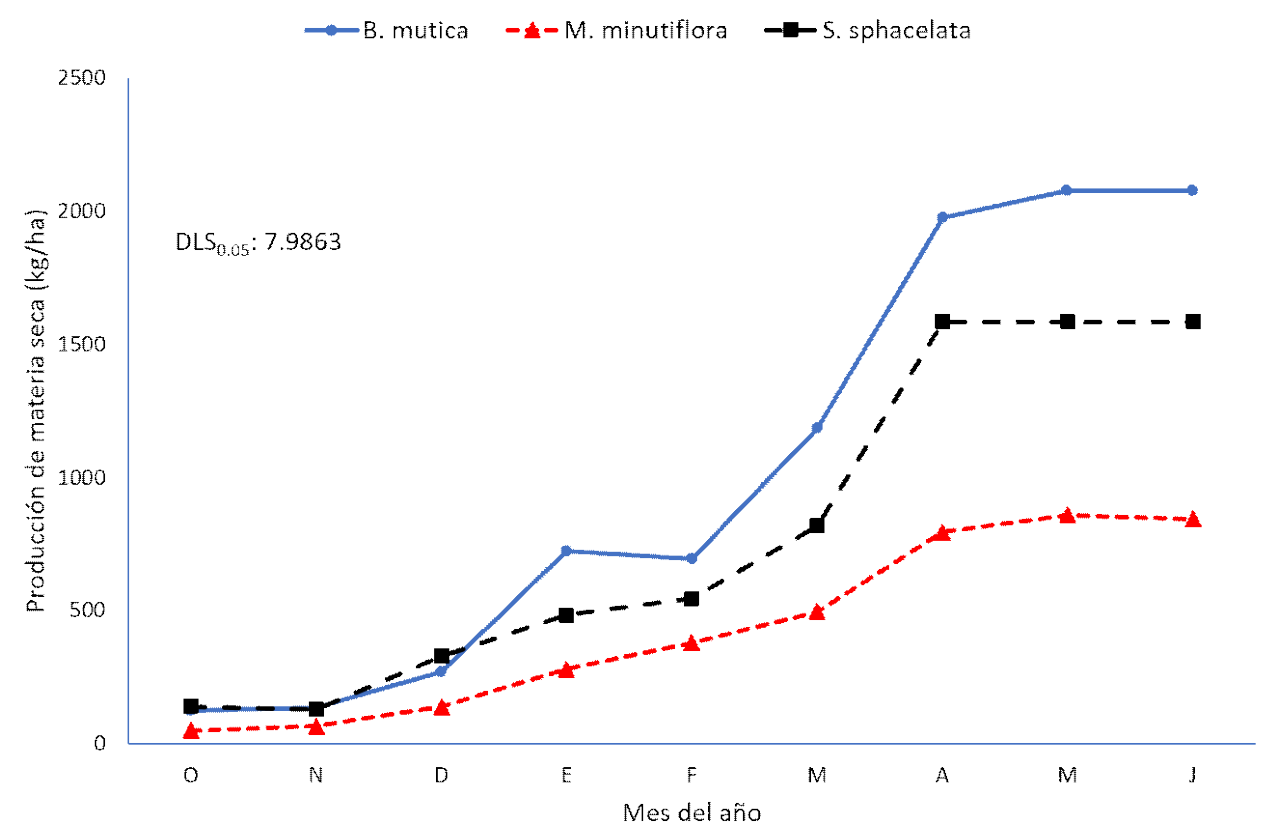

Figura 1. Fluctuaciones mensuales en rendimiento de materia seca de tres especies de gramíneas tropicales en Oxapampa, Perú

La producción de materia seca para $B$. mutica varía entre 10 y $39 \mathrm{t} \mathrm{MS} / \mathrm{ha}$ en zonas con mayor temperatura como en Yurimaguas, Perú, y con la incorporación de fertilizantes y riego (Zambrano, 1995). Calderón (2015), con una fertilización a base de estiércol de Cavia porcellus reportó rendimientos de $4.67 \mathrm{t}$ MS/ha para $S$. sphacelata al primer corte. Mayores rendimientos (15 t MS/ha) fueron reportados para $S$. sphacelata con incorporación de enmiendas cálcicas y abonos orgánicos (Juárez, 2016), así como producciones de 6-10 t MS/ha con buen manejo (Borrajo y Pizzio, 2006). Asimismo, valores de 8-16 t MS/ha (Mas, 2007) y 10-25 t MS/ha (Alvim et al., 1986; Martins et al., 2004) con fertilización nitrogenada y riego. En el caso de los rendimientos de MS de $M$. minutiflora, Hubbell (1980) reporta rendimientos de 3-8 t MS/ha, similares a los reportados en el presente estudio, mientras que producciones de $25 \mathrm{t} \mathrm{MS/ha}$ fueron reportados en ensayos similares (Martins et al., 2004).

\section{Temperatura y humedad del suelo}

La temperatura del suelo en la que se desarrolló M. minutiflora muestra un promedio significativamente más alto $\left(20.48^{\circ} \mathrm{C}\right)$ que los demás suelos que sustentan a $S$. sphacelata $\left(19.59^{\circ} \mathrm{C}\right)$ y a $B$. mutica $\left(19.58^{\circ} \mathrm{C}\right)$. Estos valores sugieren una relación inversamente proporcional con respecto a la producción de biomasa aérea, ya que $B$. mutica, se desarrolla en un suelo con menor temperatura, muestra mayor biomasa, lo que podría estar jugando un rol importante en la disminución del flujo de calor al suelo (Loch, 2004).

También se pudo apreciar que la variación en temperatura mensual del suelo que sostiene a M. minutiflora es muy pequeña, manteniéndose casi constante (Figura 2). De otro lado, la temperatura del suelo de $S$. sphacelata a partir de diciembre inicia un incremento continuo hasta abril, cuando la temperatura se estabiliza; mientras que la temperatura del suelo que sostiene a $B$. mutica 
se mantuvo estable como en el caso del suelo de M. minutiflora, pero con la diferencia de los valores de las temperaturas. Estos patrones de temperatura del suelo sugieren que en regiones cálidas es beneficioso el mantenimiento de temperaturas más frescas, como es el caso del suelo que sustenta a $B$. mutica. Este comportamiento podría estar relacionado a las demandas de evaporación y disponibilidad de agua en el suelo. En efecto, el porcentaje de humedad del suelo de la especie que tiene mayor producción de materia seca (B. mutica), tiene un promedio significativamente mayor de humedad $(26.8 \%)$ que el suelo de $S$. sphacelata (25.8\%), y M. minutiflora (25.5\%).

Los datos de temperatura y humedad muestran una relación inversamente proporcional, ya que cuanto mayor era el promedio de temperatura se registró menor humedad del suelo, probablemente debido a una mayor pérdida de agua por evaporación. La Figura 3 muestra las variaciones promedio de la humedad de los suelos, donde se puede apreciar que el suelo del M. minutiflora tiene un patrón más claro que el de las otras especies $y$, a su vez, el que menos contenido de humedad posee.

En el análisis de la influencia de la temperatura y humedad del suelo en la producción primaria en función de los resultados de altura de planta y rendimiento de materia seca durante el ciclo de vida evaluado se observó que la temperatura de los suelos que sostiene a $B$. mutica y $S$. sphacelata son similares y menores que la del pasto $M$. minutiflora. Con respecto a la humedad del suelo no se encontró diferencias estadísticas; sin embargo, se observa una tendencia en el suelo del $B$. mutica a presentar mayor contenido de humedad. Así mismo, se observó mejor cobertura de $B$. mutica, seguido de $S$. sphacelata y de $M$. minutiflora. Un buen nivel de cobertura influye positivamente, particularmente en la conservación de la humedad del suelo y la producción primaria (Zapata, 2010).
En general, la temperatura y precipitación influyen significativamente sobre la producción de biomasa de especies tropicales (Blanco, 1990; Rosemberg et al., 1990). Además, la temperatura puede afectar el desarrollo vegetativo del cultivo, inducción floral, formación y maduración de las semillas acelerando la tasa de procesos metabólicos hasta dos veces por cada $10^{\circ} \mathrm{C}$ de incremento de temperatura (Loch, 2004). La producción de biomasa aérea de las especies evaluadas estuvo aparentemente influenciada por los patrones de temperatura y humedad, observándose una relación directamente proporcional a la humedad del suelo y una aparente relación inversa con la temperatura del suelo.

\section{Progreso Fenológico y Valor Nutritivo del Forraje}

\section{Proteina cruda $(P C)$}

El contenido promedio de $\mathrm{PC}$ de $B$. mutica, $S$. sphacelata y $M$. minutiflora fue estadísticamente diferente $(\mathrm{p}<0.05)$. La mayor cantidad de PC promedio de las tres etapas fenológicas fue alcanzada por $S$. sphacelata $(7.45 \%)$, seguida por B. mutica $(6.83 \%)$ y $M$. minutiflora $(5.83 \%)$. Los estados fenológicos (Cuadro 1) influyeron significativamente sobre el contenido de PC $(\mathrm{p}<0.05)$.

El mayor porcentaje de PC en las tres especies se observó en la etapa de botón floral (B. mutica 9.4\%, S. sphacelata 9.0\%; M. minutiflora $7.0 \%$ ), disminuyendo con el avance de la madurez. No obstante, $S$. sphacelata muestra haber mantenido el mejor porcentaje de proteína (6.0\%) comparada con B. mutica y M. minutiflora que reducen en mayor magnitud el contenido de PC ( 4.35 y $3.75 \%$, respectivamente) en la etapa de maduración de semilla (Figura 4). En general, se observa que, en la etapa de botón floral y maduración de semilla, la diferencia en PC entre especies es mayor que en la etapa de inicio de floración. 
Cuadro 1. Edad de botón floral, inicio de la floración y maduración de las especies

\begin{tabular}{lccc}
\hline \multirow{2}{*}{ Especie } & \multicolumn{3}{c}{ Edad (meses) } \\
\cline { 2 - 4 } & Botón floral & Inicio de floración & Maduración de semilla \\
\hline Setaria sphacelata & 5 & 6 & 7 \\
Brachiaria mutica & 6 & 7 & 8 \\
Melinis minutiflora & 7 & 8 & 9 \\
\hline
\end{tabular}

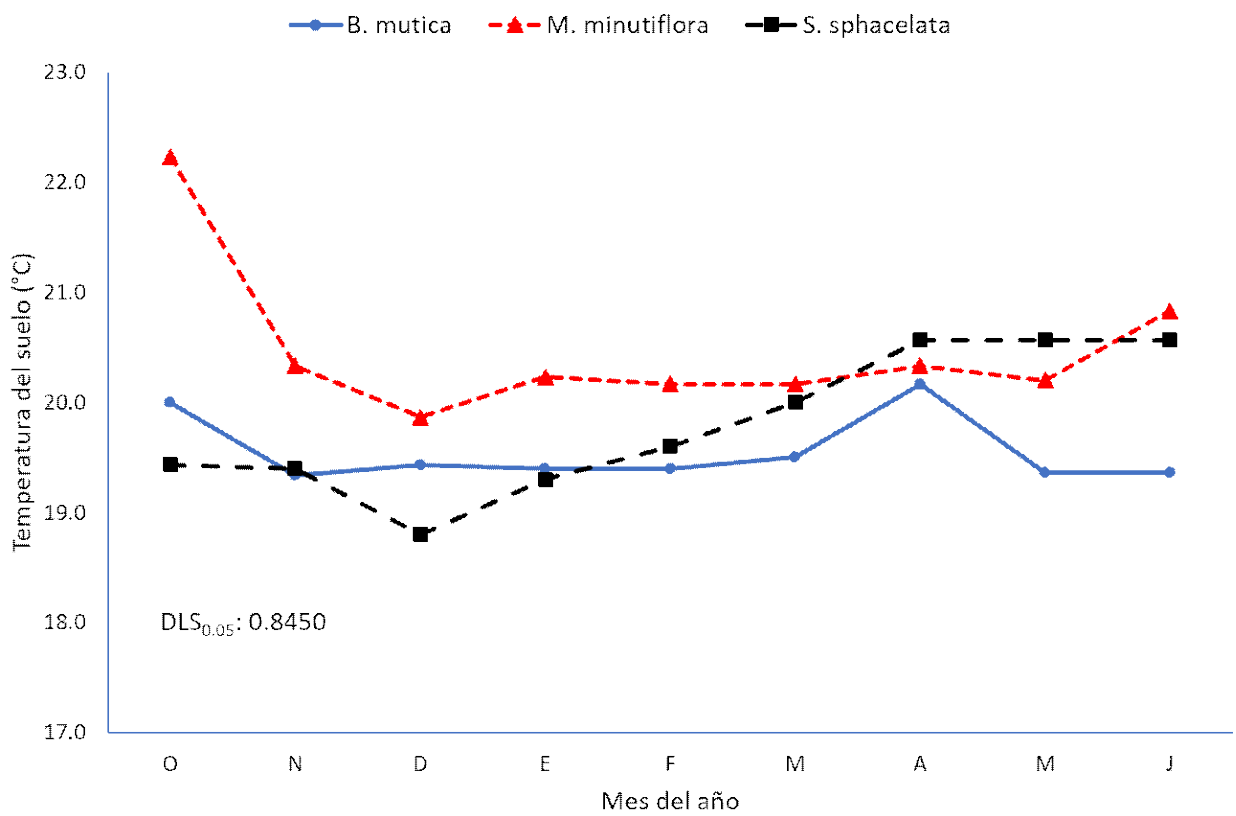

Figura 2. Variaciones mensuales de la temperatura del suelo que sustenta a tres especies de gramíneas tropicales en Oxapampa, Perú

El contenido promedio de proteína encontrado en el pasto Brachiaria, es superior a los reportados en Colombia por Valdivia et al. (1971) y Trujillo et al. (1986), con valores que varían desde $9 \%$ hasta $4.5 \%$ en los 30 y 75 días de rebrote, respectivamente. A su vez, Martins et al. (2004) reportan valores de hasta $12 \%$, pero sin indicar la edad de rebrote. En la costa peruana se reportan valores inferiores, aún con el uso de fertilización y riego en periodos de crecimiento menores a 77 días (Chauca, 1971; Abaunza et al., 1991). Estos autores coinciden al señalar que en la época de menor precipitación pluvial existe una menor concentración de proteína debido a que coincide con la etapa de maduración y acumulación de material fibroso.

Los resultados para M. minutiflora están dentro de los rangos hallados por Hubbell (1980) de 4-14\% de PC, valores que varían en función de la edad de la planta y factores abióticos. Por otra parte, Romero y Mattera (2011) reportan que el contenido de proteína varía entre 8 y $10 \%$ para $S$. sphacelata, pero en estadios vegetativos tiernos puede llegar a $15 \%$ y disminuir en estadios maduros hasta 4\%. Sánchez (2011), con fertilización a base 


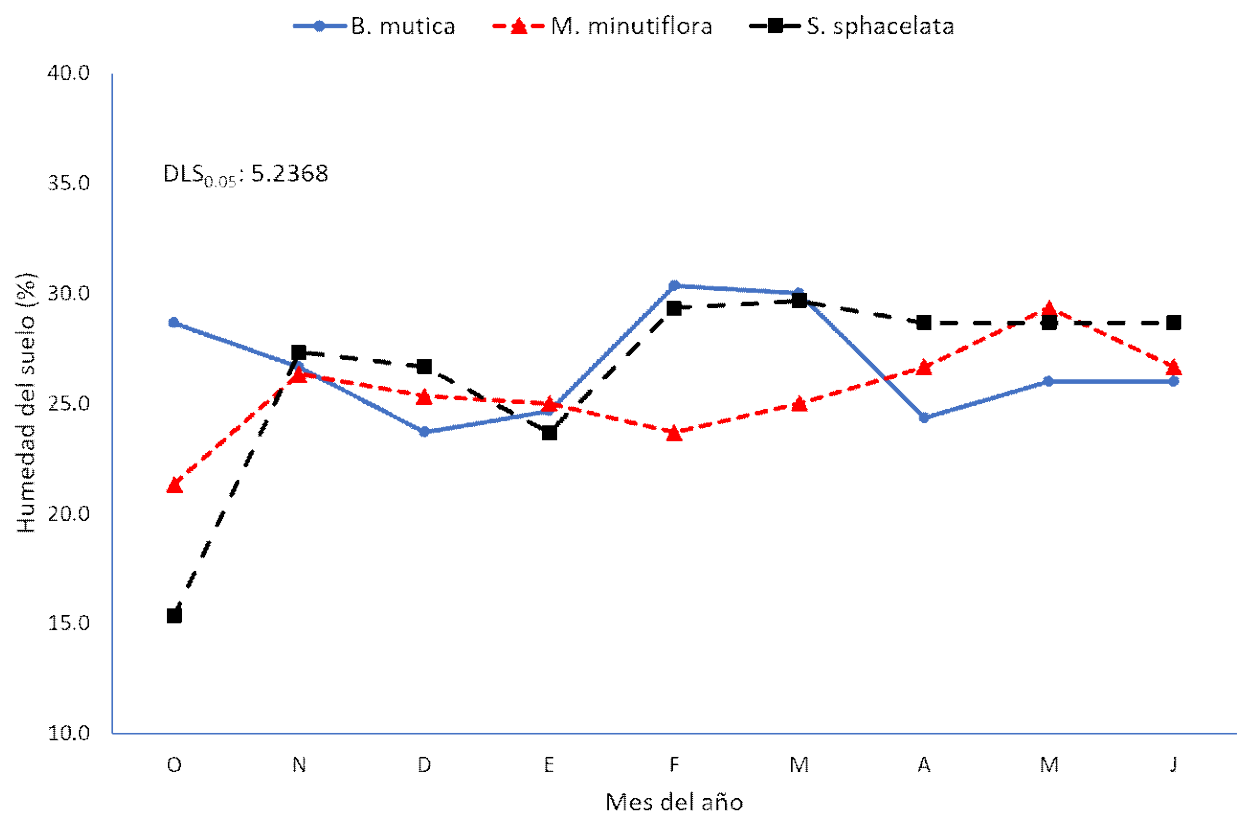

Figura 3. Fluctuaciones mensuales en la humedad del suelo que sustenta a tres especies de gramíneas tropicales en Oxapampa, Perú

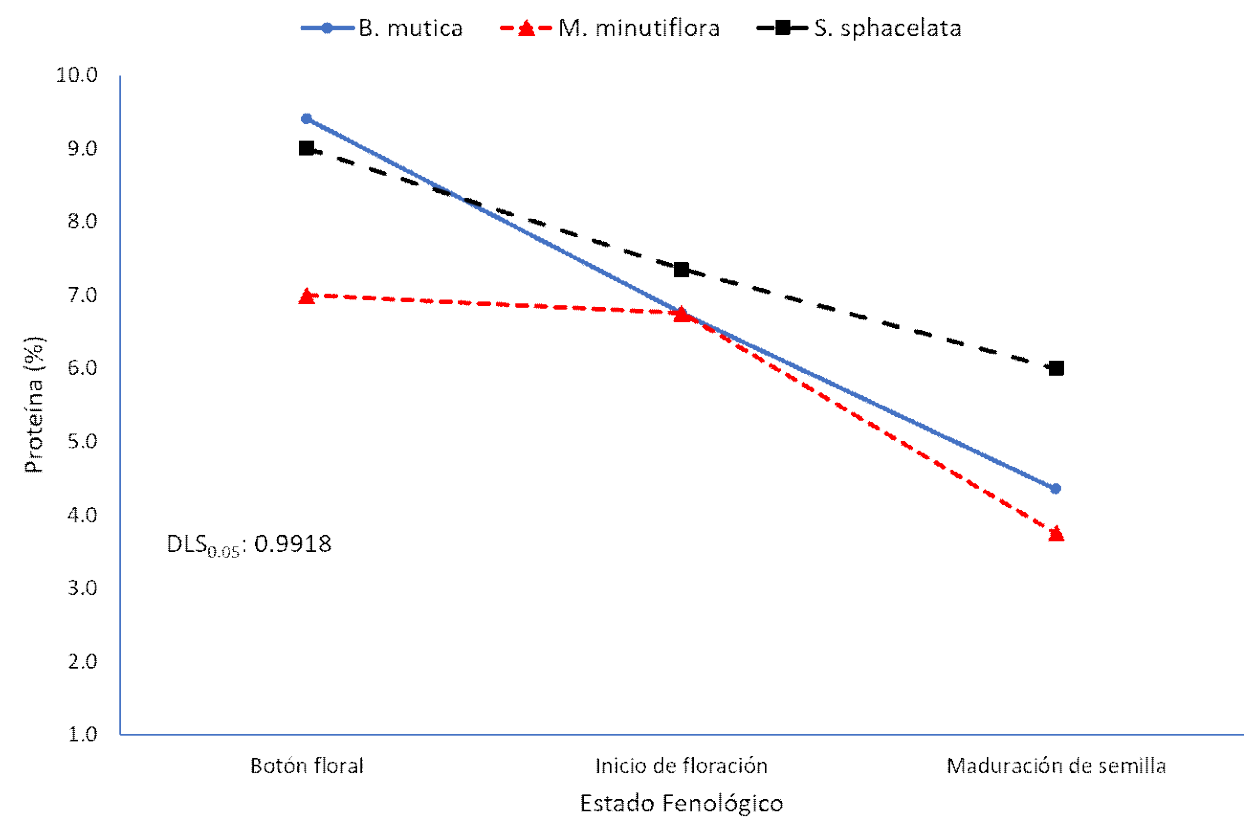

Figura 4. Variación del contenido de proteína cruda en tres estados fenológicos de tres especies de gramíneas tropicales en Oxapampa, Perú 


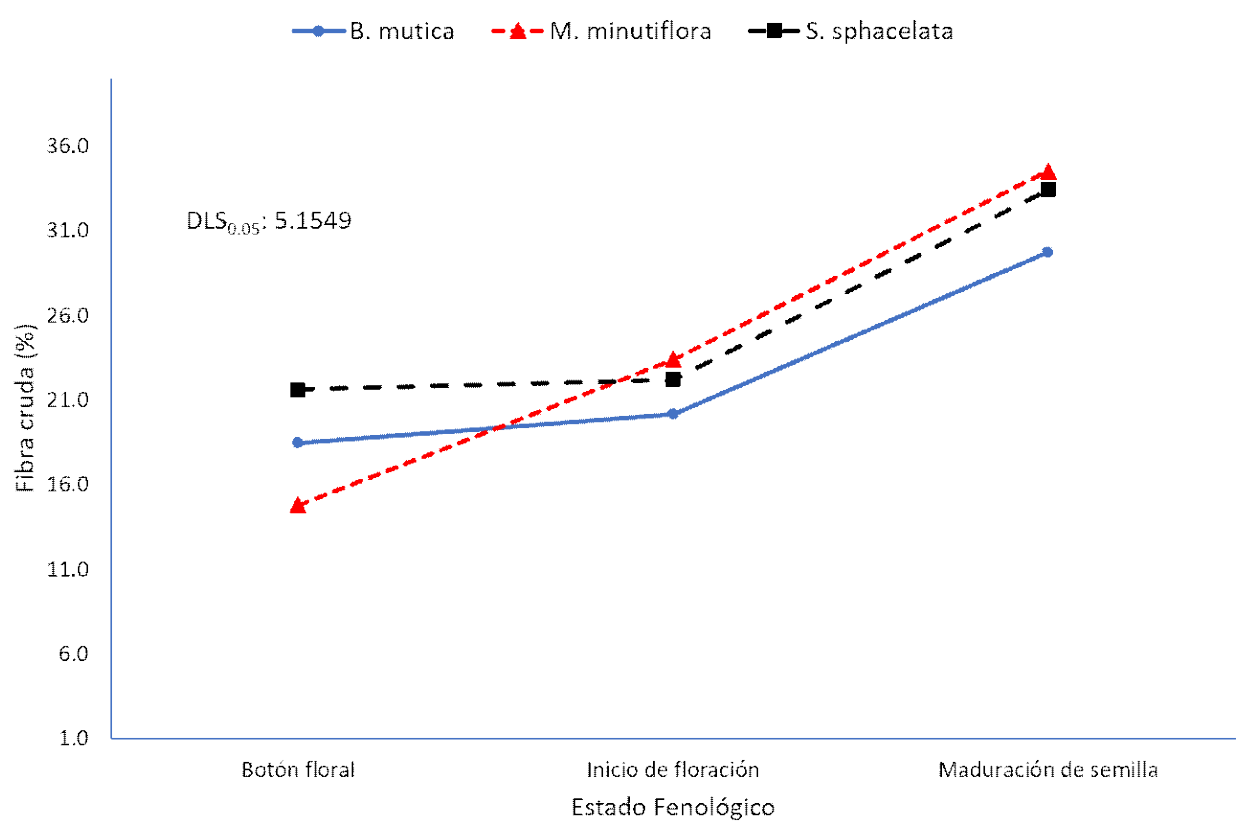

Figura 5. Efecto de estados fenológicos sobre el contenido de fibra cruda de tres especies de gramíneas tropicales en Oxapampa, Perú

de NPK en Ecuador reportó valores de 17.5, 16.3 y $15.0 \%$ de PC a los 120,135 y 150 días de corte, respectivamente. De igual forma, Gerdes et al. (2000) encontró para $S$. sphacelata que el contenido de PC de planta entera, hojas y tallos cortados a los 35 días fue de 7.5\%. En tanto, Heuzé y Trang (2017) encontraron valores de $9.1 \%$ de PC para la misma especie, mientras que Chuquimarca (2016) encuentra $14.6 \%$ de PC con la aplicación de micorrizas, demostrando el potencial nutricional de esta especie.

Fibra cruda (FC)

No se observó diferencia estadística en el contenido promedio de fibra cruda entre las gramíneas evaluadas (B. mutica $22.78 \%$, M. minutiflora $24.24 \%$, S. sphacelata 25.74\%). Las etapas fenológicas influyeron significativamente en el porcentaje de fibra acumulado $(\mathrm{p}<0.01)$. La Figura 5 muestra que $M$. minutiflora incrementa su contenido de fibra más rápidamente en comparación a $S$. sphacelata y B. mutica.
Los valores de fibra cruda encontrados en este estudio son relativamente bajos comparados con los valores de Romero et al. (2016), quien encontró $67.26 \%$ de fibra cruda en $B$. mutica en plena floración. Por su parte, Llanca et al. (2017) encontraron valores de $27.9 \%$ de fibra cruda para $S$. sphacelata. En cambio, los valores de fibra cruda del presente estudio son inferiores a los reportados de 79,80 y $85 \%$ en pasto $B$. mutica por Gutiérrez (1999), como resultado de una frecuencia de corte de 30, 60 y $90 \%$ del total de la planta en etapa madura. Sin embargo, para S. sphacelata, Sánchez (2011) reporta valores de $35.20,31.74$ y $35.87 \%$ de fibra cruda a los 120, 135 y 150 días, respectivamente.

Romero y Mattera (2011) reportan ligeros aumentos de fibra cruda en $S$. sphacelata a medida que la planta avanza en edad, mostrando valores de $30.1,31.5$, $32.2 \%$ de fibra cruda en los días 28,42 y 168 , respectivamente. Por otro lado, Heuzé y Trang (2017) reportaron $34.3 \%$ de fibra cru- 


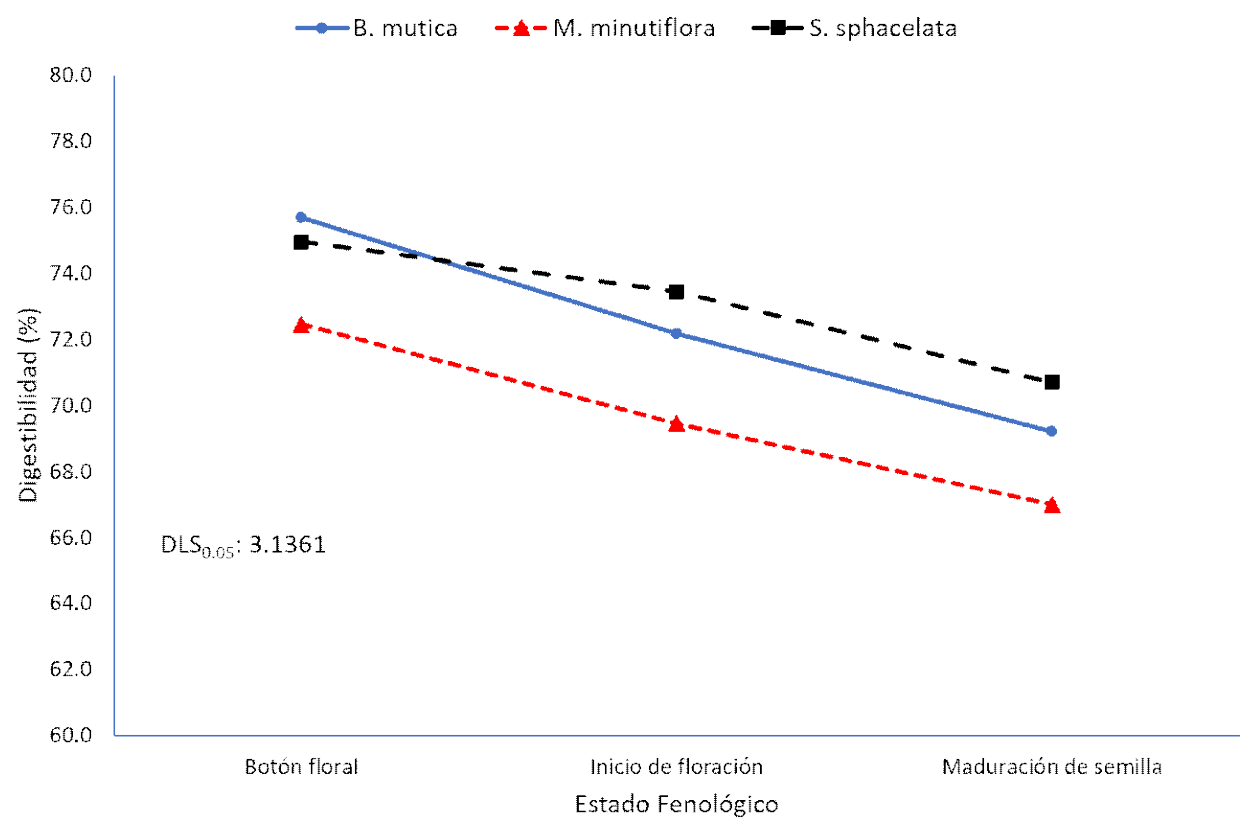

Figura 6. Efecto del estado fenológico sobre la digestibilidad de tres especies de gramíneas tropicales en Oxapampa, Perú

da en $S$. sphacelata a los 35 días de corte. Cabe resaltar que $B$. mutica incrementa en menor grado el contenido de fibra cruda.

Digestibilidad in vitro de la materia seca (DIVMS)

El porcentaje promedio de digestibilidad de las especies estudiadas muestra diferencia estadística $(\mathrm{p}<0.05)$. $S$. sphacelata y $B$. mutica mostraron porcentajes estadísticamente superiores de digestibilidad promedio que M. minutiflora. S. sphacelata mostró una digestibilidad de $73 \%$ para los tres estados fenológicos, B. mutica de $72 \%$ y $M$. minutiflora de 70\%. El análisis de la interacción de especies por estados fenológicos muestra diferencias estadísticas significativas $(p<0.05)$, donde el patrón general es de una reducción en la digestibilidad con el avance de la fenología. Los valores más bajos correspondieron a M. minutiflora, seguido del B. mutica y $S$. sphacelata (Figura 6).
Los resultados de digestibilidad de $B$. mutica en este estudio fueron superiores (72.4\%) a los valores de 58-62\% reportados por Abaunza et al. (1991). Por su parte, Martins et al. (2004) reportan valores de $70 \%$, mientras que Posada (2011) reporta valores de $55.8 \%$ en B. mutica. Por otro lado, M. minutiflora alcanzó valores de $69.6 \%$ de digestibilidad en este estudio, siendo mayores a los reportados por Peters et al. (2010) con valores de hasta $55 \%$ de digestibilidad máxima, dependiendo del estado fenológico de la planta. S. sphacelata alcanzó valores de $73.0 \%$ de digestibilidad en este estudio, siendo ligeramente inferiores a los reportados por Valdivia et al. (1971) de $75.8 \%$ a los 63-67 días de edad. Asimismo, Avellaneda (2015) encontró valores similares (78.42, 74.48 y $72.24 \%$ a los 35,45 y 55 días de edad, respectivamente). 
Los resultados de digestibilidad fueron superiores al 58-62\% hallado por Abaunza et al. (1991) y de hasta $70 \%$ en B. mutica por Martins et al. (2004). Asimismo, Posada (2011) reporta valores de $55.8 \%$ de digestibilidad para B. mutica. De otro lado, el porcentaje de digestibilidad del presente estudio fue inferior al $75.8 \%$ reportado por Valdivia et al. (1971), debido a que fue medido en plantas jóvenes de 63-77 días y en otras condiciones de clima y suelos.

En términos generales la especie con mayor valor nutritivo fue el pasto $S$. sphacelata, con de $7.45,25.7$ y $73 \%$ para proteína cruda, fibra cruda y digestibilidad in vitro de la materia orgánica respectivamente, seguido por B. mutica $(6.83,22.8$ y $72.4 \%$, respectivamente) y $M$. minutiflora (5.83, $24.2,69.6 \%$, respectivamente). Respecto a los estados fenológicos, los resultados corroboran que la calidad decrece con la edad de la planta, siendo más acelerado estos cambios en M. minutiflora.

\section{Conclusiones}

- Brachiaria mutica alcanza el mayor rendimiento de materia seca $(8978 \mathrm{~kg} / \mathrm{ha})$, seguido de Setaria sphacelata $(5755$ $\mathrm{kg} / \mathrm{ha})$ y Melinis minutiflora (4338 $\mathrm{kg} / \mathrm{ha}$ ).

- M. minutiflora es una gramínea de bajo rendimiento y calidad nutricional, pero se adapta bien a suelos secos, además contiene mayor porcentaje de materia seca y periodo de crecimiento de hasta 9 meses.

- La producción aérea muestra una relación directamente proporcional a la humedad del suelo y una aparente relación inversa con la temperatura del suelo.

- El mayor contenido de proteína cruda fue alcanzado por S. sphacelata $(7.45 \%)$, seguida de B. mutica $(6.83 \%)$ y $M$. minutiflora $(5.83 \%)$.
- Las tres especies mostraron valores similares de fibra (22.78-22.70\%), valores que se reflejaron en la digestibilidad (71-73\%).

\section{Literatura Citada}

1. Abaunza M, Lascano C, Giraldo $H$, Toledo J. 1991. Valor nutritivo y aceptabilidad de gramíneas forrajeras tropicales en suelos ácidos. Pasturas Trop 13(2): 2-8.

2. Alvim J, Botrel D, Novelly E. 1986. Produção de gramíneas tropicais e temperadas, irrigadas na época da seca. Rev Soc Brasileira Zootec 15: 384-392.

3. AOAC. 1984. Official methods of analysis. Association of Official Analytical Chemists. $12^{\text {th }}$ ed. Washington DC: Association of Official Analytical Chemists. $1141 \mathrm{p}$.

4. Avellaneda J. 2015. Comportamiento agronómico, composición química y degradabilidad ruminal in situ del pasto miel (Setaria sphacelata), Cantón Pangua provincia de Cotopaxi. Tesis de Maestría. Sangolquí: Univ. de las Fuerzas Armadas. 145 p.

5. Blanco J. 1990. Establecimiento y producción de gramíneas y leguminosas forrajeras en Peroto, Bolivia. I Reunión Red Internacional de Pastos Tropicales. Lima, Perú.

6. Bogdan V. 1997. Pastos tropicales y plantas forrajeras. México: AGT. $461 \mathrm{p}$.

7. Borrajo C, Pizzio R. 2006. Manual de producción y utilización de Setaria. Proyecto ganadero de Corrientes. $11 \mathrm{p}$. [Internet]. Disponible en: http:// www.produccionbovina.com.ar/ produccion_y_manejo_pasturas/ pasturas_cultivadas_megatermicas/178Manual_Setaria.pdf 
8. Carámbula M. 1996. Pasturas naturales mejoradas. Montevideo: Hemisferio Sur. 524 p.

9. Calderón E. 2015. Utilización de diferentes té de estiércol en la producción de Setaria sphacelata (pasto Miel). Tesis de grado. Riobamba: Escuela Superior Politécnica de Chimborazo. $138 \mathrm{p}$.

10. Costa N, Gonzales C, Da Cruz J. 1990. Introducción y evaluación de gramíneas y leguminosas forrajeras en Curo Preto D`Oeste. En: I Reunión Red Internacional de Pastos Tropicales. Lima

11. Chauca L. 1971. Diferentes niveles de fertilización e intervalos de corte y su influencia en la calidad de pasto pangola (Digitaria decumbens Stent). Tesis de Ingeniero Zootecnista. Lima: Univ. Nacional Agraria La Molina. $71 \mathrm{p}$.

12. Chuquimarca E. 2016. Efecto de diferentes niveles de micorriza más la adición de una base estándar de humus en la producción primaria forrajera de la Setaria aphacelata (pasto miel). Tesis de Ingeniero Zooetecnista. Chimborazo: Escuela Superior Politécnica de Chimborazo. $101 \mathrm{p}$.

13. Cuadrado H, Torregroza L, Jiménez N. 2004. Comparación bajo pastoreo con bovinos machos de ceba de cuatro especies de gramíneas del género Brachiaria. MVZ Córdoba 9: 438-443.

14. Gerdes L, Werner J, Colozza M, Possenti R, Schammass E. 2000. Avaliação de características de valor nutritivo das gramíneas forrageiras marandu, setária e Tanzânia nas estações do ano. Rev Bras Zootec 29: 955-963. doi: 10.1590/S1516-35982000000400003

15. Gutiérrez BC. 1999. Efectos de la intensidad y frecuencia de corte y rendimiento y valor nutritivo de $B$. mutica. Tesis de Maestría. Lima: Univ. Nacional Agraria La Molina. $66 \mathrm{p}$.

16. Harvard-Duclos B. 1969. Las plantas forrajeras tropicales. España: Ed. Blume. $380 \mathrm{p}$.
17. Heuzé V, Trang G 2017. Setaria gigante (Setaria sphacelata var. splendida). Instituto Nacional de Investigación Agronómica. Fedipedia. [Internet]. Disponible en: http://www.feedipedia.org/ node $/ 380$

18. Hoffmann A, Lucatelli M, Silva J, Azeuedo $N$, Marinho $S$, Albuquerque M, Lopes A, Moreira P. 2004. Impact of the invasive alien grass Melinis minutiflora at the savanna forest ecotone in the Brazilian Cerrado. Diversity Distrib 10: 99-103.

19. Hubbell D. 1980. Técnicas agropecuarias aplicadas a zonas tropicales. México: Ed Trillas. 369 p.

20. Imbaquingo E, Naranjo D. 2010. Comportamiento inicial de aliso (Alnus nepalensis D. Don) y cedro tropical (Acrocarpus fraxinifolius Wight y Arn), asociados con brachiaria (Brachiaria decumbens Stapf) y pasto miel (Setaria sphacelata (Schumach) Stapf y CE Hubb). Tesis de Ingeniero Forestal. Univ. Técnica del Norte. $63 \mathrm{p}$.

21. Ira J. 1979. Handbook of tropical forage grasses. New York: Garland SIPM Press. $101 \mathrm{p}$.

22. [IUCN] Unión Internacional para la Conservación de la Naturaleza. 2016. Restauración funcional del paisaje rural: manual de técnicas. Serie técnica: gobernanza forestal y economía 3: $284 \mathrm{p}$.

23. Juárez C. 2016. Efecto de dos enmiendas cálcicas y dos abonos orgánicos en el rendimiento de la especie forrajera Setaria sphacelata nicarion, Molino-pampa-Chcachapoyas-Amazonas 2015. Tesis de grado. Chachapoyas: Univ. Nacional Toribio Rodríguez de Mendoza. $93 \mathrm{p}$.

24. León R. 2008. Pastos y forrajes producción y manejo. $2^{\circ}$ ed. Quito, Ecuador: EdicionesCientíficas Agustín Álvarez. $251 \mathrm{p}$. 
25. Llamas O, Leonhard A, Yfrán M, Pereira M, Bernardis C, Fernández A. 2017. Evaluación de la fertilización nitrogenada sobre la producción y el valor nutritivo de Setaria sphacelata cv. Narok. Agrotecnia 24: 17-21.

26. Llanca L, Pérez H, Mestanza C, López R, Quintana J. 2017. Influencia de los sistemas de producción y pisos altitudinales en la composición bioquímica y rendimiento del pasto nicarión (Setaria sphacelata). Rev Invest Agroprod Sustentable 1(2): 9-16.

27. Loch D. 2004. Seed formation, development, and germation in tropical and subtropical species. Wallingford, Oxon, UK: CAB International. $89 \mathrm{p}$.

28. Martínez M. 1971. Calibración y comparación de algunos métodos para la determinación de humedad del suelo. Tesis de Maestría, Chapingo, México: Univ. Nacional Autónoma de Chapingo. $120 \mathrm{p}$.

29. Martins C, Leonel L, Haridasan M. 2004. Capim-gordura (Melinis minutiflora P. Beauv.), uma graminea exótica que compromete a recuperacao de áreas degradadas em unidades de conservacao. R Árevore Vicosa-MG 28: 739-743.

30. Mas C. 2007. Setaria sphacelata. Una gramínea a tener en cuenta. Rev INIA 10: 33-36.

31. NuñezJ. 2017. Perfil alimentario y plan de pastoreo para la producción lechera con pasturas Panicum maximum Jacq. Tesis de Maestría. Lima: Univ. Nacional Agraria La Molina. $47 \mathrm{p}$.

32. Peters M, Franco L, Schmidt A, Hincapié B. 2010. Especies forrajeras multipropósito: Opciones para productores del trópico americano. Cali, Colombia: CIAT. $213 \mathrm{p}$.

33. Posada R. 2011. Comparación de dos métodos in vitro para estimar la digestibilidad de pastos tropicales en rumiantes. Rev Citecsa 2(2): 13-24.
34. Ramírez O, Hernández A, Carneiro $S$, Pérez J, Francisco J, Raymuno A, Guadalupe J, Cervantes A. 2009. Acumulación de forraje, crecimiento y características estructurales del pasto Mombaza (Panicum maximum Jacq.) cosechado a diferentes intervalos de corte. Téc Pec Mex 47: 203-213.

35. Romero O, Romero P, Hernández G, Morales G. 2016. Brachiaria mutica como alternativa en la alimentación del ganado bovino en el Rancho Santa Elena Palizada, Campeche. Tesis de grado. México: Univ. Autónoma Agraria Antonio Narro. 45 p.

36. Romero J, Mattera J. 2011. Rendimiento y calidad del forraje de forrajeras megatérmicas bajo distintas frecuencias de defoliación. Ganadería Técnicos.

37. Rosemberg M, Malpartida E, Gomez A. 1990. Establecimiento y producción de gramíneas y leguminosas forrajeras en Satipo, Junín-Perú. I Reunión Red Internacional de Evaluación de Pastos Tropicales, Lima, Perú.

38. Sánchez J. 2011. Establecimiento de una pradera de Setaria splendida (Setaria sphacelata) para corte, en la finca Punzara de la Universidad Nacional de Loja. Tesis de Maestría. Loja: Univ. Nacional de Loja. 93 p.

39. Tilley M, Terry A. 1963. A two-stage technique for the in vitro digestion of forage crops. Grass Forage Sci 18: 104111. doi: 10.1111/j.1365-2494.1963.tb00335. $\mathrm{x}$

40. Trujillo G, Posada J, Sierra O. 1986. Efecto de la edad de rebrote en la calidad nutritiva de $B$. decumbens. Pasturas Trop 8: 7-9.

41. Trujillo A, Uriarte G. 2011. Valor nutritivo de las pasturas. Departamento de producción animal y pasturas. Universidad de la República, Uruguay. [Internet], [15 mayo de 2014]. Disponible en: http:/ /prodanimal.fagro.edu.uy 
42. Valdivia R, Barua J, Clavo N, Campos L, Villaroel C. 1971. Energía digestible y nivel de consumo de forrajes tropicales. En: II Reunión de la Asociación Latinoamericana de Producción Animal - ALPA. Colombia.

43. Zambrano R. 1995. Informe sobre pastos y forrajes. Lima: Ministerio de Agricultura.

44. Zapata A. 2010. Efecto del guácimo (Guazuma ulmifolia), carao (Cassia grandis) y roble (Tabebuia rosea) sobre la productividad primaria neta aérea y composición florística de pasturas naturales en Muy Muy y Matiguás, Nicaragua. Tesis de Maestría. Turrialba, Costa Rica: Centro Agronómico Tropical de investigación y Enseñanza. 153 p.

45. Zuloaga O, Morrone O, Davidse G. Filgueiras S, Peterson M, Soreng J, Judziewicz J. 2003. Setaria. In: Catalogue of New World Grasses (Poaceae): III. Subfamilies Panicoideae, Aristidoideae, Arundinoideae, and Danthonioideae. Contr U.S. Natl Herb 46: 569-593. 